\title{
Degradation of transparent conductive oxides; Mechanistic insights across configurations and exposures
}

\author{
Heather M. Lemire ${ }^{a, b}$, Kelly A. Peterson ${ }^{a}$, Samuel Sprawls ${ }^{b}$, Kenneth Singer ${ }^{b}$, Ina T. Martin ${ }^{b}$ \\ , Roger H. French ${ }^{a}$ \\ ${ }^{a}$ SDLE Center; ${ }^{b}$ MORE Center \\ Case Western Reserve University, 10900 Euclid Ave, Cleveland, OH, USA
}

\begin{abstract}
Understanding transparent conductive oxide (TCO) degradation is critical to improving stability and lifetime of both organic and inorganic thin film PV modules, which utilize TCOs, like indium tin oxide (ITO), aluminumdoped zinc oxide (AZO) and fluorine-doped tin oxide (FTO) as electrodes. These TCOs must retain their long-term functionality in diverse outdoor environments. In addition to bulk material degradation, interfacial degradation, a frequent avenue for failure in PV systems, is promoted by exposure to environmental stressors such as irradiance, heat and humidity. ITO, AZO and FTO samples in an open-faced configuration were exposed to damp heat and ASTM G154 for up to 1000 hours. The effect of exposure on electrical and optical properties and surface energies of cleaned samples was measured. Yellowness, haze, water contact angle and resistivity of the different materials trended differently with exposure time and type, indicating the activation of distinct degradation mechanisms.

An encapsulated configuration study was conducted on ITO and AZO, exposing samples to the above accelerated exposures and two outdoor exposures (1x suns and 5x suns on a dual axis trackers), with and without PEDOT:PSS layers. PEDOT:PSS increases the yellowness and haze of ITO and AZO, but does not accelerate the increase in resistivity, suggesting that the optical and electrical degradation mechanisms are not coupled. Additionally, the hazing/roughening mechanism of PEDOT:PSS on AZO appears to be photo-sensitive; 5x outdoor exposure samples demonstrated distinctly higher haze than damp heat exposed samples.
\end{abstract}

Keywords: Transparent Conductive Oxides, Degradation, Accelerated and Outdoor Exposures

\section{INTRODUCTION}

Photovoltaics (PV), display and touch screens, organic light emitting diodes (OLEDs) and other opto-electronic devices, utilize transparent conductive oxides (TCOs). Attaining a high conductivity while maintaining optical transparency makes them ideal electrical contacts in these systems. However, when deployed in the field for long term applications, durability concerns arise. The cost and reliability of solar power are often cited as a primary limiting factors for its widespread adoption. These aspects of PV power systems are linked; increasing the lifetime of an installation can reduce the levelized cost of energy (LCOE, the total cost of the installation levelized over its lifetime). Many components in PV systems require lifetime extension; in particular, TCOs (transparent conductive oxides) are a critical failure mode in many PV technologies that hold the potential to be cost-effective. Delamination at the TCO-absorber and TCO-glass interfaces have been reported in thin film silicon solar modules ${ }^{123}$, CIGS modules ${ }^{456}$ and OPV technologies ${ }^{78910}$. An increase in resistivity and structural changes are observed in the TCO bulk 6111213 . Corrosion is known to occur across a number of optoelectronic devices $^{1214}$. In significantly degraded CIGS cells, replacing the TCO restores its electrical activity to near peak performance ${ }^{4}$.

The chemically sensitive TCO surfaces can be modified with a thin polymer layer, silanization (resulting in covalent bond formation), and the chemisorption of small molecules, among other methods ${ }^{15}$. PEDOT:PSS (poly(3,4-ethylenedioxythiophene) poly(styrenesulfonate)) is a polymer commonly used as an electron blocking

Further author information: (Send correspondence to H.M.L.)

H.M.L.: E-mail: heather.lemire@case.edu, Telephone: 12163680135 
layer in OLEDs and standard architecture OPV devices. Unfortunately, PEDOT:PSS is highly acidic, causing corrosion of the TCO layer ${ }^{891016}$.

In this study, three commercially available TCO materials on glass were exposed to two types of accelerated environmental exposures and two outdoor exposures in one of 3 configurations. Degradation was characterized by monitoring changes in their optical and electrical properties and surface free energies.

The accelerated exposures used here are industry qualification testing standards. The intention of an accelerated exposure is to provide rapid, straight-forward insights into the lifetime performance of a material or device. However, recent studies and colloquial knowledge have called into question the reasonableness of these exposures and their ability to accurately predict lifetime. For example, a single stressor exposure is straight-forward to conduct and will likely cause degradation. When environmental stressors are combined, a more difficult exposure to conduct, different degradation mechanisms will appear. Thus, a reasonable accelerated exposure is defined as one which induces the same degradation mechanisms as seen in outdoor exposures. To address the above, in this study, a variety of accelerated and outdoor exposures were conducted, the properties of the TCO monitored, and insights into a variety of mechanisms of TCO degradation gathered from these exposures and the comparisons between accelerated and outdoor exposures.

\section{EXPERIMENTAL PROCEDURE}

ITO (Colorado Concept Coatings LLC), AZO (Zhuhai Kaivo Electronic Components Co., Ltd.), and FTO (Hartford Glass Company Inc.) on glass were purchased commercially. Samples were cleaned before collecting contact angle data (before and after the accelerated exposure). Sample cleaning involved a series of $10 \mathrm{~min}$ sonications in $30{ }^{\circ} \mathrm{C}$ solvents (acetone, methanol and isopropanol), drying under nitrogen gas, and a $15 \mathrm{~min}$ UV ozone clean at $60{ }^{\circ} \mathrm{C}$ (Novascan PSDP-UV8T). Note that gentle scrubbing with an optical wipe was used after the IPA sonication to remove strongly adhered surface species after various exposures.

Four exposure protocols were used in this study, 2 accelerated, 2 real world; ASTM G154 (cyclic), IEC 61626 Damp Heat (DH), and outdoor exposures at $1 \mathrm{x}$ and $5 \mathrm{x}$ concentrations on a dual-axis tracker (see Table 1). Samples were exposed in one of 3 configurations; open-faced, edge seal encapsulated, and edge seal encapsulated with PEDOT:PSS. The encapsulation was accomplished by wet etching the ITO and AZO samples with $50 \%$ $\mathrm{HCl}$ solution, applying the cleaning procedure, applying a $1 / 3$ of $10 \mathrm{~mm}$ width of Quanex edge seal, followed by lamination in a mini module laminator at $90{ }^{\circ} \mathrm{C}$ and $500 \mathrm{mPa}$ after $10 \mathrm{~min}$ evacuation. Samples were subjected to exposures for up to the standard $1000 \mathrm{~h}$ in $168 \mathrm{~h}$ (1 week) increments. After each exposure step, 2-3 samples were removed, cleaned, characterized and stored.

Table 1. Exposure parameters used in this study.

\begin{tabular}{|c|c|c|}
\hline Exposure Name & Conditions & Equipment \\
\hline ASTM G154 Cycle 4, "cyclic" & $\begin{array}{c}8 \mathrm{~h} @ 70{ }^{\circ} \mathrm{C}, 1.55 \mathrm{~W} / \mathrm{m}^{2} / \mathrm{nm} @ 340 \mathrm{~nm}, \\
4 \mathrm{~h} \mathrm{@} 50{ }^{\circ} \mathrm{C} \text { with spray in dark }\end{array}$ & QUV Spray \\
\hline IEC 61626, "damp heat", "DH" & $85{ }^{\circ} \mathrm{C}, 85 \%$ relative humidity & CSZ ZPH8 \\
\hline Real World 1x & Cleveland weather June-August & dual-axis tracker \\
\hline Real World 5x & $\begin{array}{c}\text { Cleveland weather June-August, } \\
\text { front surface mirror concentrator }\end{array}$ & dual-axis tracker \\
\hline
\end{tabular}

To quantify the properties of the TCO, optical, electrical and surface measurement techniques were used. The surface free energy (SFE) was calculated from contact angles with water and diiodomethane using the Kruss EasyDrop, DSA4 system and the Wu method. Contact angle values are the average and standard deviation of five 50 frame video measurements on 1-2 samples; SFEs are calculated from these contact angles. Transmission spectra were obtained using a Varian Cary $6000 \mathrm{i}$. Percent haze, yellowness index, $\mathrm{L}^{*}, \mathrm{a}^{*}$, and $\mathrm{b}^{*}$ values were obtained using a Hunterlab UltraScan Pro Colorimeter. The resistivity of the ITO films was measured using an EDTM R-chek four point probe (which is prone to edge effects deviating up to 20\%), and the resistivity of 
the AZO and FTO films was measured using a Keithley 2400 Source and Lucas Labs four point probe (which penetrates slightly into the film). Reported error bars are the standard deviation of the mean where 3 or more data points were available, or approximate standard deviations of the mean drawn from comparable trials or baseline measurement variation on the instrument. Microscope images were collected with a Leica DM 2500M microscope at 5x magnification.

\section{RESULTS AND DISCUSSION}

No statistically significant degradation was observed in FTO during the open-faced configuration study. The stability of this TCO is consistent with prior work ${ }^{2311}$. Statistically significant degradation is defined as a correlation coefficient with time of greater than 0.7 and/or data having visual trending which coincides with domain science knowledge. Because of FTOs stability, it was excluded from the encapsulation study.

\subsection{Mechanisms in Different Accelerated Exposures}

In this section, we will consider the open-faced damp heat and cyclic exposures. Figures 1 and 2 compare statistically significant variables in the damp heat and cyclic exposures for ITO. In both exposures, there appears to be an an initial step change in the resistivity, water contact angle, and haze after the first 168 hours of exposure. This step increase suggests some initial changes to the material, possibly surface rearrangements, which then stabilize.
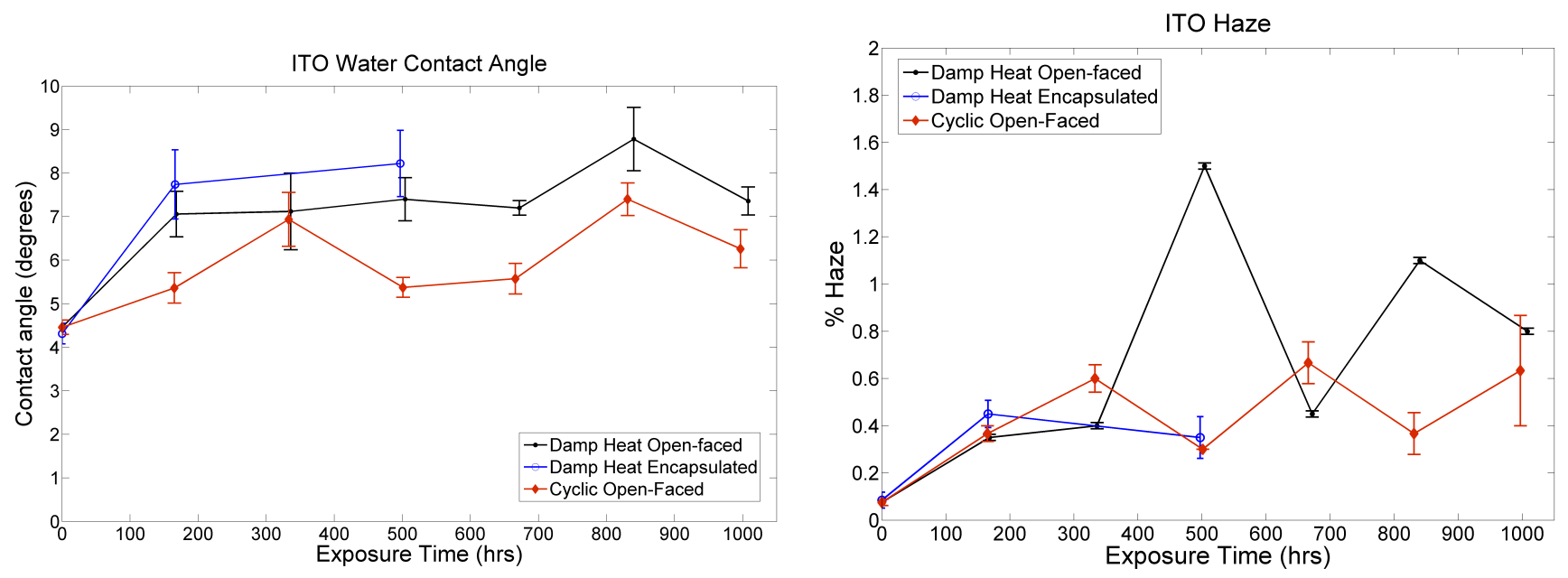

Figure 1. Water contact angle and haze of ITO in damp heat and cyclic exposures

In contrast to the ITO, AZO resistivity increases differently in damp heat and cyclic exposures; open-faced damp heat increases resistivity rapidly, possibly exponentially, while the cyclic and encapsulated damp heat samples increase in a linear fashion (Figure 3). In contrast, the optical properties of AZO (taken in the center of the sample) are more degraded by the cyclic than by the damp heat exposure; both yellowness index and percent haze show steady increases in cyclic exposure (Figure 4). These results indicate the activation of two different degradation mechanisms; a water-sensitive and a photo-induced. This conclusion is consistent with the literature, which suggests that water absorption at the grain boundaries increases carrier scatter and therefore resistance but does not affect the optical properties ${ }^{5111213}$.

It should be noted that both open-faced exposures also caused delamination-type edge effects in AZO; for damp heat exposure (Figure 5 on the left) edge effects measure approximately $3 \mathrm{~mm}$ wide on a $5 \mathrm{~cm}$ square sample at 1000 hours of exposure. The right of figure 5 compares the damp heat and cyclic edge effects, demonstrating that cyclic exposure appears to result in complete removal of the TCO at the TCO-glass interface, while the damp heat exposure appears to be partially through delamination. 


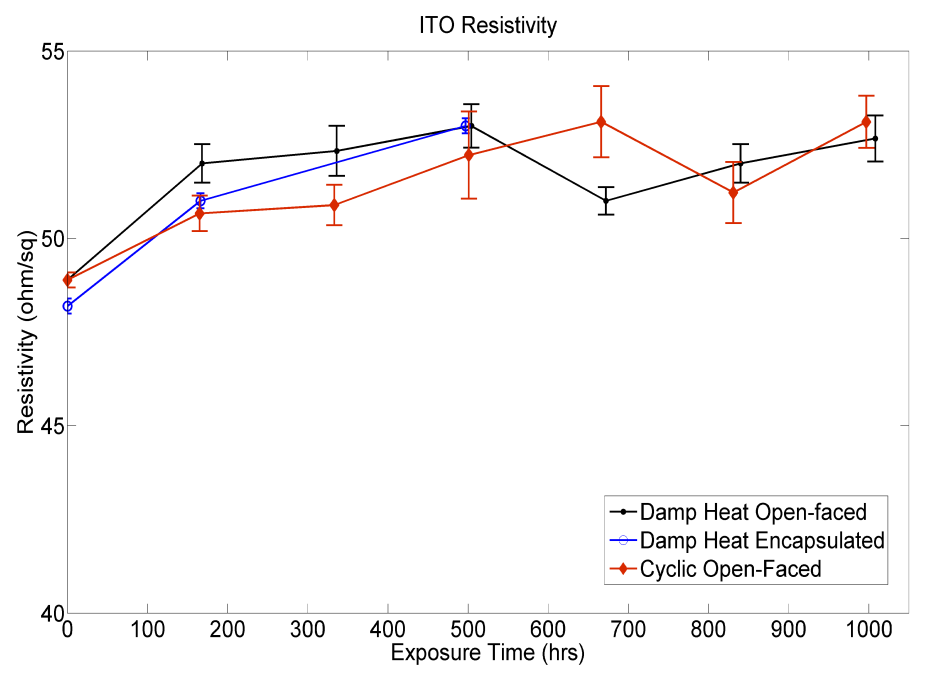

Figure 2. Resistivity of ITO in damp heat and cyclic exposures

The cyclic exposure of both TCOs maintains a lower water contact angle than the damp heat exposure, which could be due to the exposure chamber (acting as a form of UV-ozone cleaning) or an indication of surface changes.

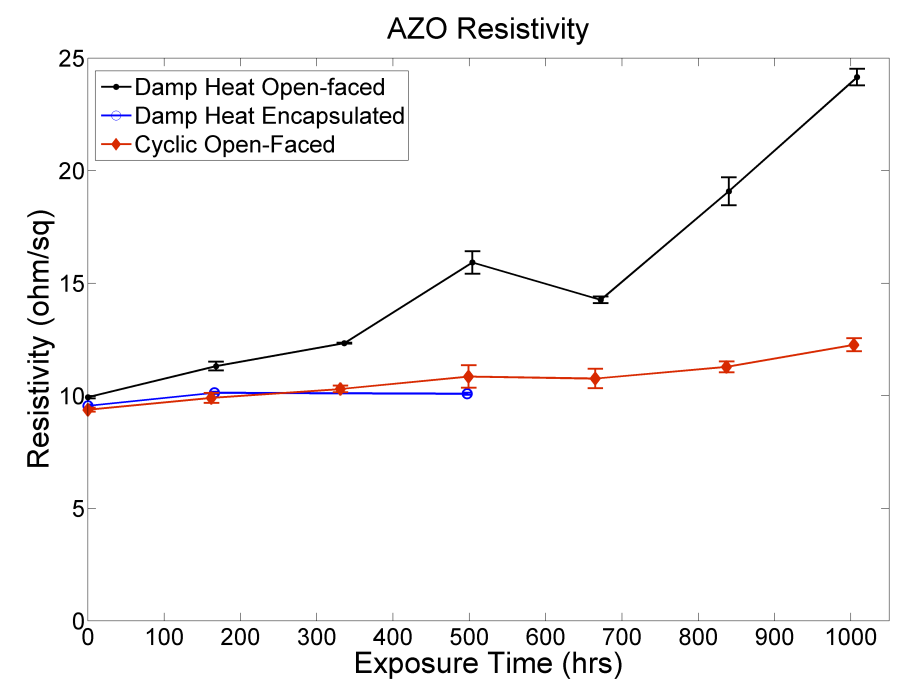

Figure 3. Resistivity ofAZO in damp heat and cyclic exposures

\subsection{Encapsulated TCO Degradation: The effects of PEDOT:PSS}

Knowing that different exposure types cause different degradation mechanisms, it is also important to examine different exposure configurations, such as placing the TCO in a device context, to observe interactions with other layers during degradation. A layer of PEDOT:PSS, which is known to damage ITO ${ }^{891016}$, incorporated into a glass-glass edge-seal only lamination forms a first approximation of the environment a TCO used in an OPV device would see.

ITO encapsulated with PEDOT:PSS in damp heat has a higher yellowness index than open-faced damp heat exposure, seen in Figure 6, which could indicate the beginning of a degradation mechanism. However, the 

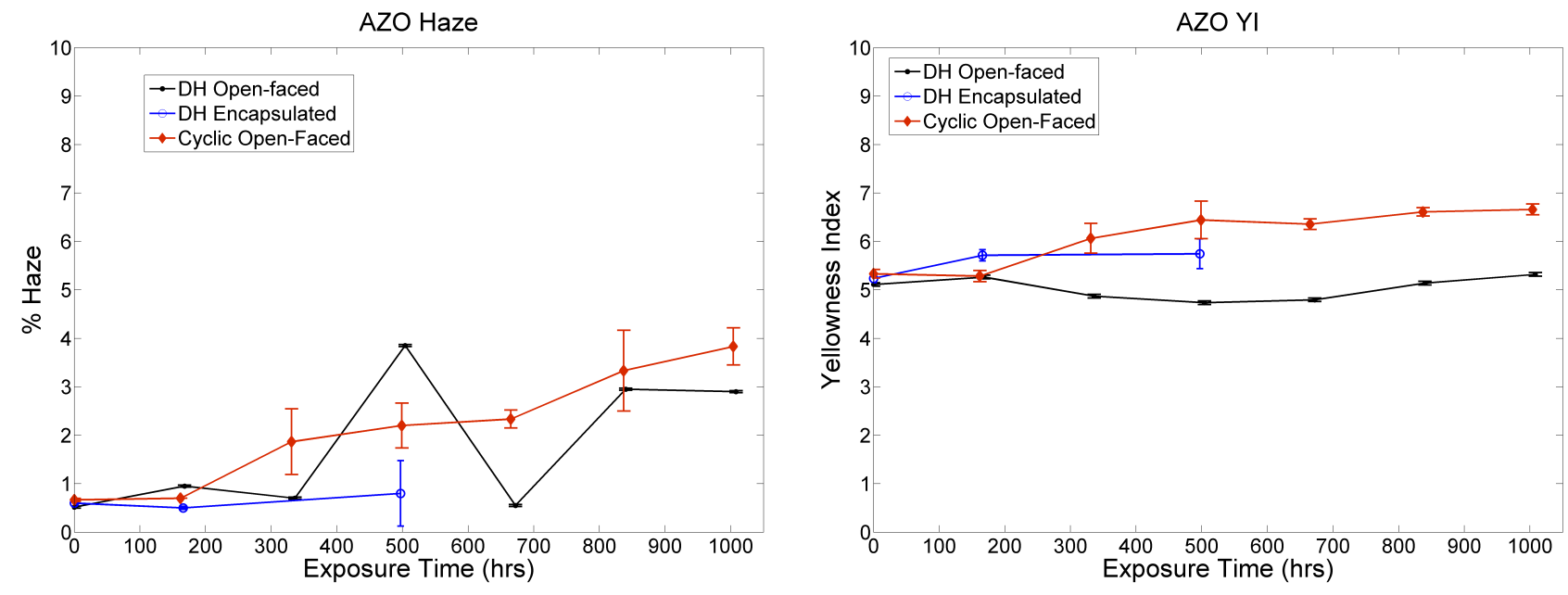

Figure 4. Haze and Yelloness index of AZO in damp heat and cyclic exposures
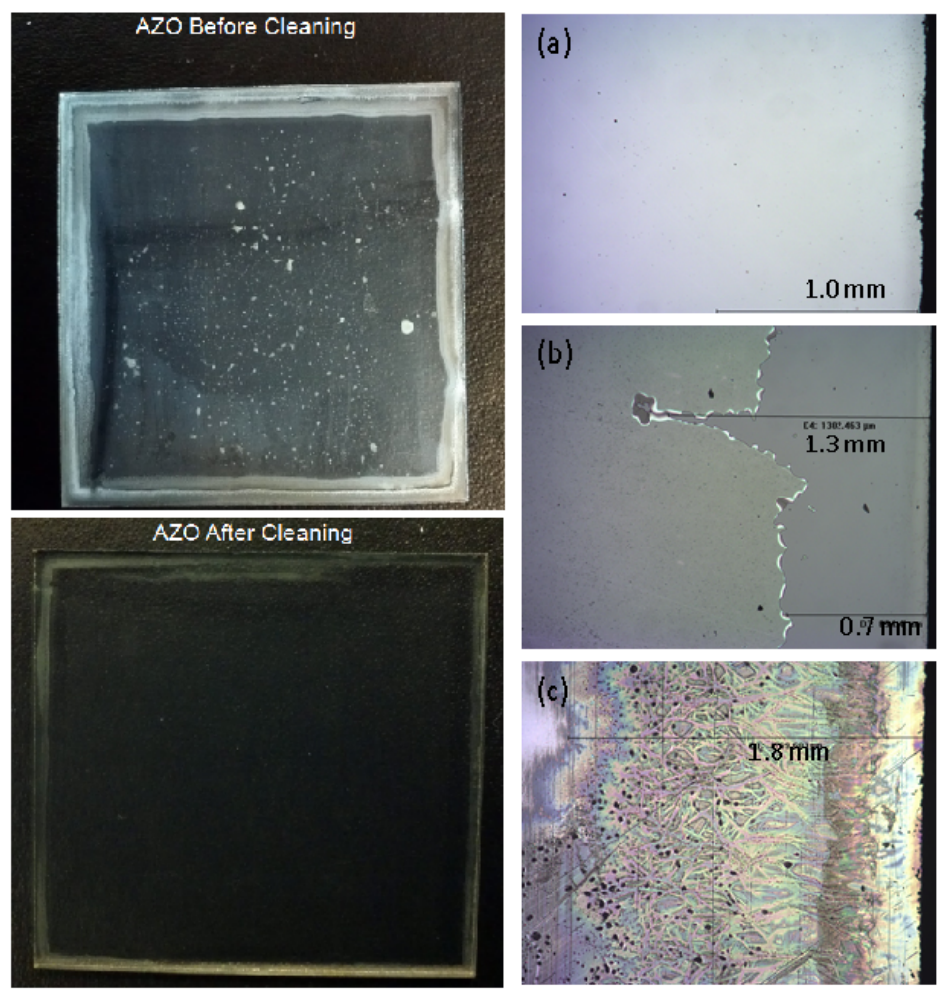

Figure 5. Edge effects in Damp Heat exposed AZO. Left side: damp heat exposure at 1000 hours; the sample is $5 \mathrm{~cm}$ square. Right Side: 5x magnification microscope images of the edge of AZO on glass (a) as recieved, (b) after $1000 \mathrm{~h}$ cyclic, and (c) after $1000 \mathrm{~h}$ damp heat exposure.

PEDOT:PSS does not accelerate the resistivity increase of ITO in damp heat.

The application of PEDOT:PSS demonstrates greater degradation to AZO than ITO. AZO exposure also results in an increase in yellowness index, as seen in Figure 7. Additionally, AZO has a higher haze, a lower water contact angle (Figure 8), and a decrease in the transmission spectra and smoothing of the interference fringes (data not shown). These indicate a roughening of the AZO surface, which is also known to occur without 
ITO YI

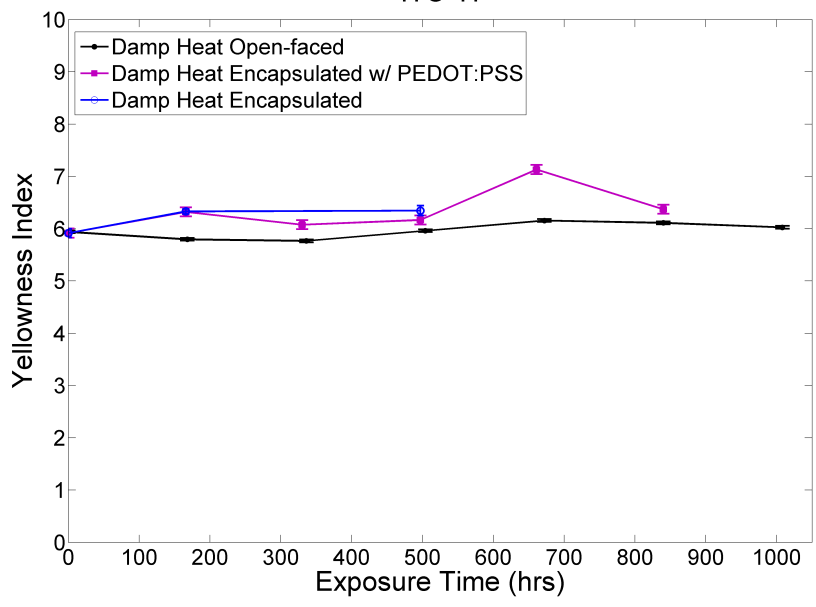

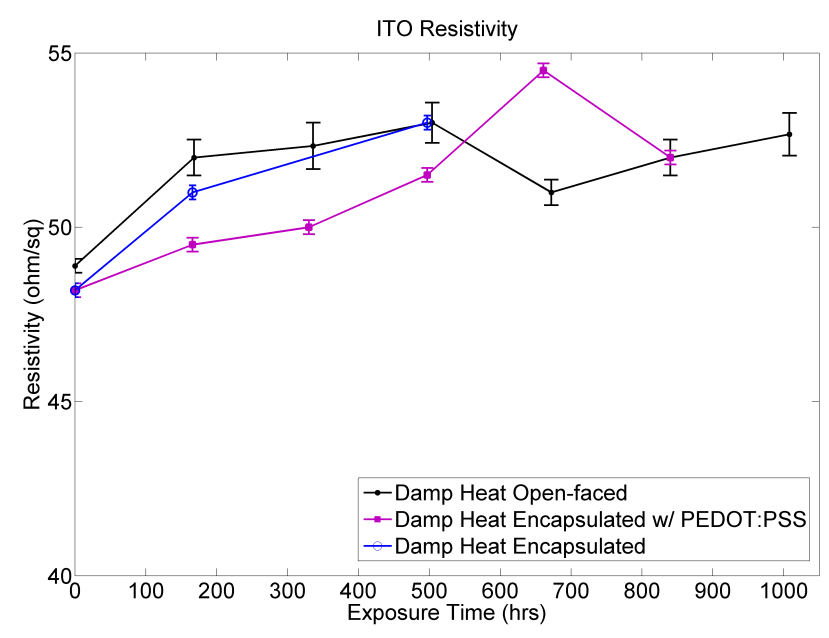

Figure 6. Effect of PEDOT:PSS layer and aging on ITO yelloness index and resistivity.

the presence of PEDOT:PSS ${ }^{6113}$.

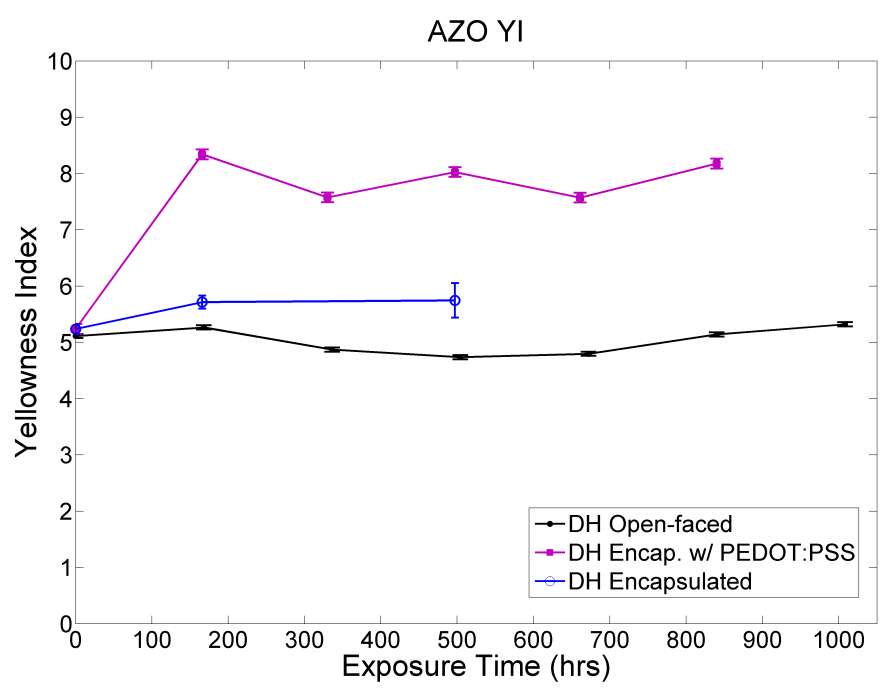

Figure 7. Effect PEDOT:PSS layer and aging on AZO yellowness index.

Furthermore, when AZO encapsulated with PEDOT:PSS is exposed to 5x concentration outdoors, the haze is distinctly higher than damp heat exposed encapsulated samples, as seen in Figure 9. This implies that the hazing mechanism is accelerated by light or UV radiation.

However, this photo-induced mechanism does not appear to be related to the resistivity increase mechanism, as the outdoor $5 \mathrm{x}$ exposure resistivity is comparable to that of the damp heat exposure, showing no extra acceleration.

\section{CONCLUSIONS}

In this study, AZO, ITO and FTO were exposed to several types of accelerated and outdoor exposures. FTO was found to be the most stable TCO, followed by ITO, where AZO is extremely susceptible to environmental degradation. The electrical properties are the most susceptible to humidity, while the optical properties are susceptible 

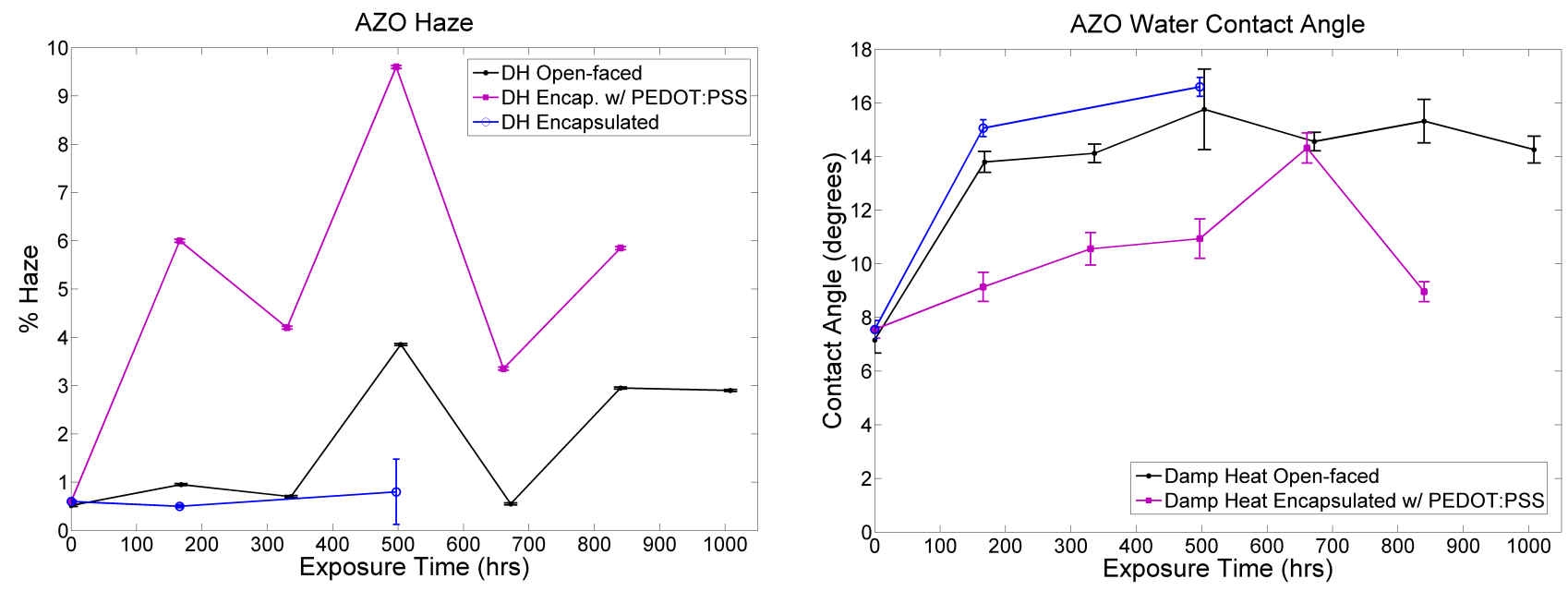

Figure 8. Effect PEDOT:PSS layer and aging on AZO haze and water contact angle.
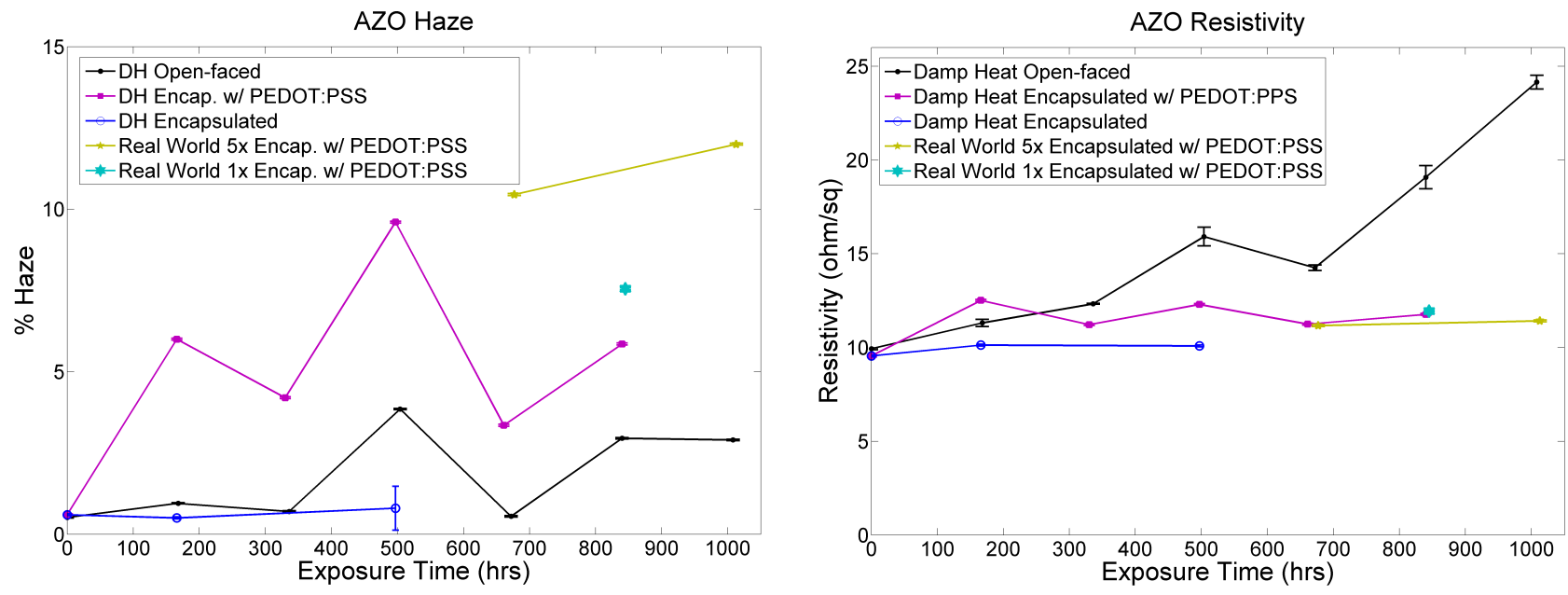

Figure 9. Haze and resistivity changes in AZO in various exposures and configurations, suggesting photo-sensitive mechanism.

to UV exposure, indicating that these are two separate degradation mechanisms. The inclusion of PEDOT:PSS in the exposure configuration causes an increase in yellowness and haze beyond changes caused by just environmental stressors, indicating an additional degradation mechanism with the addition of PEDOT:PSS. These PEDOT:PSS-induced mechanisms appear to be photo-sensitive, as exposure to 5x suns significantly increased the haze.

The application of an interfacial silane layer has also been considered for preventing damage to TCOs from environmental stressors. Studies seem to indicate silanes have promise for reducing edge effects in damp heat exposed AZO. For the full analysis of the effect of silane interfacial layers on the degradation of TCOs, please see upcoming AVS proceedings paper "Interfacial Layer Engineering of Transparent Conductive Oxides for Optoelectronic Device Application".

\section{ACKNOWLEDGMENTS}

Funding for the encapsulated TCO study was from the Bay Area Photovoltaic Consortium Prime Award No. DE-EE0004946, Subaward Agreement No. 60220829-51077-T. Funding for the open-faced TCO study was from 
Underwriters Laboratories. Research was performed at the SDLE Center at Case Western Reserve University, which was established through funding through the Ohio Third Frontier, Wright Project Program Award Tech 12-004. Sample tracking and data management support was provided by REDCap? funded by the Clinical and Translational Science Collaborative (CTSC) grant support (1 UL1 RR024989 from NCRR/NIH).dy.

\section{REFERENCES}

1. Osterwald, C. R., McMahon, T. J., del Cueto, J. A., Adelstein, J., and Pruett, J., "Accelerated stress testing of thin-film modules with sno2: F transparent conductors," in [National Center for Photovoltaics and Solar Program Review Meeting Denver, Colorado March], 24-26 (2003).

2. Jansen, K. W., Varvar, A., Twesme, E., Berens, T., and Dhere, N. G., "Design of high-reliability low-cost amorphous silicon modules for high energy yield," (2008).

3. Beyer, W., Hüpkes, J., and Stiebig, H., "Transparent conducting oxide films for thin film silicon photovoltaics," Thin Solid Films 516(2), 147-154 (2007).

4. Sundaramoorthy, R., Pern, F. J., DeHart, C., Gennett, T., Meng, F. Y., Contreras, M., and Gessert, T., "Stability of tco window layers for thin-film cigs solar cells upon damp heat exposures: part ii," in [SPIE Solar Energy + Technology], 74120J-74120J, International Society for Optics and Photonics (2009).

5. Kempe, M. D., Terwilliger, K. M., and Tarrant, D., "Stress induced degradation modes in cigs minimodules," in [Photovoltaic Specialists Conference, 2008. PVSC'08. 33rd IEEE], 1-6, IEEE (2008).

6. Feist, R., Rozeveld, S., Mushrush, M., Haley, R., Lemon, B., Gerbi, J., Nichols, B., Nilsson, R., Richardson, T., and Sprague, S., "Examination of lifetime-limiting failure mechanisms in cigss-based pv minimodules under environmental stress," in [Photovoltaic Specialists Conference, 2008. PVSC'08. 33rd IEEE], 1-5, IEEE (2008).

7. Wong, K. W., Yip, H. L., Luo, Y., Wong, K. Y., Lau, W. M., Low, K. H., Chow, H. F., Gao, Z. Q., Yeung, W. L., and Chang, C. C., "Blocking reactions between indium-tin oxide and poly (3, 4-ethylene dioxythiophene): poly (styrene sulphonate) with a self-assembly monolayer," Applied physics letters 80(15), 2788-2790 (2002).

8. Jong, M. D., Ijzendoorn, L. J. V., and Voigt, M. J. A. D., "Stability of the interface between indium-tin-oxide and poly (3, 4-ethylenedioxythiophene)/poly (styrenesulfonate) in polymer light-emitting diodes," Applied Physics Letters 77(14), 2255-2257 (2000).

9. Girtan, M. and Rusu, M., "Role of ito and pedot: Pss in stability/degradation of polymer: fullerene bulk heterojunctions solar cells," Solar Energy Materials and Solar Cells 94(3), 446-450 (2010).

10. Grossiord, N., Kroon, J. M., Andriessen, R., and Blom, P. W., "Degradation mechanisms in organic photovoltaic devices," Organic Electronics 13(3), 432-456 (2012).

11. Pern, J. and Noufi, R., "An investigation of stability issues of zno and mo on glass substrates for cigs solar cells upon accelerated weathering and damp heat exposures," in [DOE SETP Review Meeting, Denver, CO], 4, 17-19 (2007).

12. Greiner, D., Gledhill, S., Köble, C., Krammer, J., and Klenk, R., "Damp heat stability of al-doped zinc oxide films on smooth and rough substrates," Thin Solid Films 520(4), 1285-1290 (2011).

13. Tohsophon, T., Hüpkes, J., Calnan, S., Reetz, W., Rech, B., Beyer, W., and Sirikulrat, N., "Damp heat stability and annealing behavior of aluminum doped zinc oxide films prepared by magnetron sputtering," Thin solid films 511, 673-677 (2006).

14. Leung, W., Chan, Y., and Lui, S., "A study of degradation of indium tin oxide thin films on glass for display applications," Microelectronic Engineering 101(0), 1 - 7 (2013).

15. Brumbach, M. and Armstrong, N. R., "Modification of transparent conducting oxide (tco) electrodes through silanization and chemisorption of small molecules," Encyclopedia of Electrochemistry (2007).

16. Allen, C. G., Baker, D. J., Albin, J. M., Oertli, H. E., Gillaspie, D. T., Olson, D. C., Furtak, T. E., and Collins, R. T., "Surface modification of zno using triethoxysilane-based molecules," Langmuir 24(23), 13393-13398 (2008). 